\title{
Primary and secondary creep in aluminum alloys as a solid state transformation
}

\author{
R. Fernández, ${ }^{1, a)}$ G. Bruno, ${ }^{2}$ and G. González-Doncel ${ }^{1}$ \\ ${ }^{1}$ Department of Physical Metallurgy, Centro Nacional de Investigaciones Metalúrgicas (CENIM) C.S.I.C., \\ Av. de Gregorio del Amo No. 8, E-28040 Madrid, Spain \\ ${ }^{2}$ Federal Institute for Materials Research and Testing (BAM), Unter den Eichen 87, D-12200 Berlin, Germany
}

(Received 28 June 2016; accepted 10 August 2016; published online 24 August 2016)

\begin{abstract}
Despite the massive literature and the efforts devoted to understand the creep behavior of aluminum alloys, a full description of this phenomenon on the basis of microstructural parameters and experimental conditions is, at present, still missing. The analysis of creep is typically carried out in terms of the so-called steady or secondary creep regime. The present work offers an alternative view of the creep behavior based on the Orowan dislocation dynamics. Our approach considers primary and secondary creep together as solid state isothermal transformations, similar to recrystallization or precipitation phenomena. In this frame, it is shown that the Johnson-Mehl-AvramiKolmogorov equation, typically used to analyze these transformations, can also be employed to explain creep deformation. The description is fully compatible with present (empirical) models of steady state creep. We used creep curves of commercially pure Al and ingot AA6061 alloy at different temperatures and stresses to validate the proposed model. Published by AIP Publishing.

[http://dx.doi.org/10.1063/1.4961524]
\end{abstract}

\section{CREEP OF METALS}

Creep of metals is a key topic for different industries such as energy, transport, or chemical. The creep behavior of pure metals and alloys has been extensively investigated in the past. This behavior is usually described through the accumulated plastic deformation $\varepsilon(t)$ as a function of time $t$ under given conditions of applied stress, $\sigma$, and temperature, $T$. Three regions are typically distinguished, namely, a primary creep region (where $\ddot{\varepsilon}(t)<0$ ); a secondary regime, also called steady state (where $\ddot{\varepsilon}(t)=0$ ); and a tertiary region (where $\ddot{\varepsilon}(t)>0$ ) before creep rupture occurs (double dot denotes second time derivative). Many authors, as Evans, ${ }^{1}$ reduce the steady state regime to a single turning point from the primary to the tertiary regions in the creep curve. This is, then, called the minimum creep rate state. For simplicity, however, we will refer throughout this work to a steady state region. Most of the investigations aimed at correlating the creep behavior with microstructural parameters of the alloy under study restrict their analysis to the steady state regime, where the creep rate is independent of time, i.e., one can write $\dot{\varepsilon}=\dot{\varepsilon}_{s s}(\sigma, T)$ (dot denotes first time derivative). ${ }^{2-4}$

From the large amount of steady state data available, there is general agreement on the following two findings: First, a power law relationship between steady state creep rate, $\dot{\varepsilon}_{s s}$, and applied stress $\sigma$ commonly holds, i.e.,

$$
\dot{\varepsilon}_{S S}=C\left(\frac{\sigma}{G}\right)^{n},
$$

where $n$ is the stress exponent, $G$ is the shear modulus, and $C$ is a microstructure and temperature dependent constant. The

\footnotetext{
a) Author to whom correspondence should be addressed. Electronic mail: ric@cenim.csic.es. Tel. +34 915538900 ext. 230.
}

exponent $n$ is differently interpreted in the existing literature: for Sherby, ${ }^{5}$ the value of $n$ establishes the dominant deformation mechanism, while Blum ${ }^{6}$ relates it to the microstructural features; $C$ presents an Arrhenius-type dependence with temperature

$$
C=K \exp \left(\frac{-Q_{c}}{R T}\right),
$$

where $Q_{c}$ is the activation energy for creep, $R$ is the universal gas constant, and $K$ is a dimensionless microstructuredependent constant. Some authors use the elastic modulus, $E$, instead of $G$, but the relation between $\left(\frac{\sigma}{G}\right)$ and the microstructure invites to use naturally Equation (1). ${ }^{6}$

Second, the activation energy for creep, $Q_{\mathrm{c}}$, is usually identified with the activation energy for atomic selfdiffusion, $Q_{\mathrm{d}}$, revealing the importance of the diffusion phenomenon in the creep process. Using $Q_{\mathrm{d}}$ in Eq. (2) implies that the term $K$ needs to contain $D_{0}$ (the pre-exponential term of the diffusion coefficient, $\left.D=D_{0} \cdot \exp \left(\frac{-Q_{d}}{R T}\right)\right)$. This is very clearly shown in the work of Sherby, ${ }^{7}$ in which data of about twenty pure metals, ranging from indium to tungsten, show a linear correlation between $Q_{\mathrm{c}}$ and $Q_{\mathrm{d}}$. However, the description of creep in metals solely based on steady state regime data represents a very limited vision of the whole phenomenon. This limitation undermines our capability to describe the creep deformation evolution with time and to predict this behavior on the basis of the material's microstructural parameters (and of the experimental conditions).

Some authors have attempted approaches to describe the creep strain evolution with time. Among the most ancient models is the one proposed by Garofalo ${ }^{8}$ in 1965 . This model restricts the description of creep evolution to the primary region $^{8}$ and writes the strain as a function of time as 


$$
\varepsilon(t)=\varepsilon_{0}+\varepsilon_{T}\left(1-e^{-v t}\right),
$$

$\varepsilon_{0}$ being the initial elastic strain, $\varepsilon_{\mathrm{T}}$ the limiting transient creep deformation, and $v$ a constant. Equation (3) requires the initial creep data in order to find appropriate fitting parameters for predicting the primary creep curve under different conditions. Clear correlations between the constant $v$ and microstructural parameters are missing in this model.

Almost 20 years later, Wilshire ${ }^{9}$ developed a modified model, which includes an additional term into the Garofalo equation, accounting for tertiary creep. This model describes creep curves by using four parameters: $\theta_{1}, \theta_{2}, \theta_{3}$, and $\theta_{4}$ combined in the following equation:

$$
\varepsilon=\theta_{1}\left(1-e^{-\theta_{2} t}\right)+\theta_{3}\left(e^{\theta_{4} t}-1\right) .
$$

The first term describes the primary and onset of secondary creep, while the second term, corresponding to the tertiary region, completes the creep curve until material failure. The model was constructed with the aim of describing complete creep curves. The model uses this description for life assessment prediction purposes. While the model is simple and elegant, $\theta_{1}$ to $\theta_{4}$ are purely fitting parameters without any microstructural/physical-base justification. Again, creep data are required to find the specific $\theta_{i}$ values. Further attempts at correlating these parameters with the physical phenomena governing the creep process have systematically failed. ${ }^{10}$

A different approach to describe the evolution of creep strain with time was developed by Mecking and Kocks, ${ }^{11}$ in their model known as "One microstructural parameter." They assumed that the microstructural evolution during plastic deformation of metallic materials can be described in terms of the evolution of the dislocation population. The deformation rate results, then, from the balance between the accumulation and annihilation of dislocations (responsible for plastic deformation). The ratio of accumulation/ annihilation for small grain size or dispersion strengthened materials can be described according to Mecking and Kocks $^{11}$ as

$$
\frac{d \rho}{d \varepsilon}=k_{1}-k_{2} \rho
$$

where $\rho$ is the dislocation density and $k_{1}$ and $k_{2}$ are the fitting parameters corresponding to the dislocation multiplication and annihilation processes, respectively. In spite of the sound physical base of this model, the above fitting parameters are again obtained from previous creep tests. ${ }^{12}$

Creep evolution has successfully been treated in the Laplace-domain for piezoelectric materials. ${ }^{13}$ This allowed fitting, without a microstructural/physical base, the strain dependence on time, and corrected the unwanted strain drift.

In summary, there is so far no physical based predictive model of creep of metals and alloys that describes the creep deformation evolution with time, even the primary regime, on the basis of only microstructural parameters.

The objective of this paper is to give a description of primary and secondary creep under the viewpoint of solid state transformations, but using the movement of dislocations as the basic mechanism. We will see that experimental data fully support this view, and the model allows estimating microstructural parameters such as the subgrain size.

\section{SOLID STATE ISOTHERMAL REACTIONS}

Solid state isothermal reactions can be divided in homogeneous and heterogeneous. ${ }^{14}$ In the first case, the probability of the transformation to occur is the same for all locations in the system, and the transformation rate decreases monotonically with time. Heterogeneous transformations are characterized by a nucleation, growth, and saturation sequence, which presents a maximum transformation rate at times $t>0$, and are usually modeled by the Johnson-MehlAvrami-Kolmogorov (JMAK) equation ${ }^{15}$

$$
f=\left(1-e^{-B t^{m}}\right)
$$

where $f$ is the fraction transformed at time $t$ during the isothermal reaction, $B$ is a mechanism dependent parameter, and $m$ is an exponent that depends mainly on geometrical and dimensional aspects. The parameter $B$ is usually described as

$$
B=\omega e^{\frac{-H}{R T}}
$$

where $\omega$ is the frequency of the process, i.e., proportional to the reaction rate, $H$ is the activation energy of the process, $R$ is the universal gas constant, and $T$ is the temperature. Usually, the parameters $B$ and $m$ are extracted by a least square fit of $\ln (1-f)$ vs. $\ln t$ data.

The JMAK equation successfully describes the kinetics of crystallization, chemical reactions, and precipitation phenomena in solid state physics. ${ }^{14,15}$ It has also been applied in other fields of physics, such as crystallization kinetics of lipids ${ }^{16}$ or polymers, ${ }^{17}$ the analysis of depositions on surface science, ${ }^{18}$ the evolution of ecological systems, ${ }^{19}$ and even in cosmology. ${ }^{20}$

\section{SOLID STATE TRANSFORMATION CREEP (SSTC) MODEL}

As mentioned before, creep is a diffusion-controlled process. This fact allows us to describe the creep strain evolution with time as an isothermal process based on atoms self-diffusion under the viewpoint of phase transformations. This can be done if creep strain is considered as a state function of time, stress, and temperature, related to the fraction of the "transformed quantity," $f$

$$
\varepsilon=A * f=A *\left(1-e^{-B t^{m}}\right),
$$

where the deformation $A$ is a function of $\sigma, T$, and microstructure of the material.

The parallelism between the main features of solid state creep transformation under isothermal conditions and precipitation, traditionally described by the JMAK equation, is displayed in Table I.

The isothermal solid state transformation creep represents the transformation of the material by creep under the action of a stress field from the initial undeformed state $\varepsilon_{0}$ (sample with length $l_{0}$ ) to the final deformed one, $\varepsilon$ (sample 
TABLE I. Comparison of precipitation and creep phenomenon in the JMAK theory.

\begin{tabular}{lcc}
\hline \hline & Precipitation & Creep \\
\hline Driving element & Vacancies & Vacancies \\
Driven element & Solute atoms & Dislocations \\
Driving force & Chemical potential & Stress \\
Nucleation preformed & Yes & Yes \\
Nuclei existing & Yes & Yes \\
& Solute atoms & Grain boundaries and \\
JMAK (m exponent) & & grain interior \\
Energy barrier & $1-3$ & 1 and 3 \\
\hline \hline
\end{tabular}

with length $l$ ) (see Figure 1). This is described for a uniaxial stress test by

$$
d \varepsilon=\varepsilon-\varepsilon_{0}=\frac{d l}{l_{0}} .
$$

Strain results from the cooperative movement of a huge amount of dislocations inside grains. This is described by Orowan's equation ${ }^{6}$

$$
\varepsilon_{O r}=M^{-1} b \rho h(g),
$$

where $b$ is the Burger's vector; $\rho$ is the mobile dislocation density, depending on applied stress; $h(g)$ is the average distance that dislocations travel inside grains/subgrains (being $g$ the grain size); and $M$ is the Taylor factor, allowing for texture. Therefore, the movement of one dislocation by the Burger's vector can be considered as a "quantum" of strain. Adding up "quanta" produces a macroscopic strain when the whole dislocation population moves within the grains of a polycrystalline metal (e.g., during creep). In other words, the cooperative movement of dislocations induces a macroscopic strain, and the fraction of strain (material "transformed by creep") increases with time. We propose that dislocation movement, solely impeded by dislocation structures as grain boundaries or dislocation forests, andlor the formation of subgrains, Blum, ${ }^{6}$ are analogous to a homogeneous solid state transformation, which has $m=1$ (in Eq. (8), see Mittemeijer ${ }^{14}$ ). This description matches the traditional consideration of creep deformation as a homogeneous phenomenon (over the whole specimen). However, during dislocation motion within the grains, subgrains start to develop in class $\mathrm{M}$ alloys, i.e., those which creep behavior is similar to pure

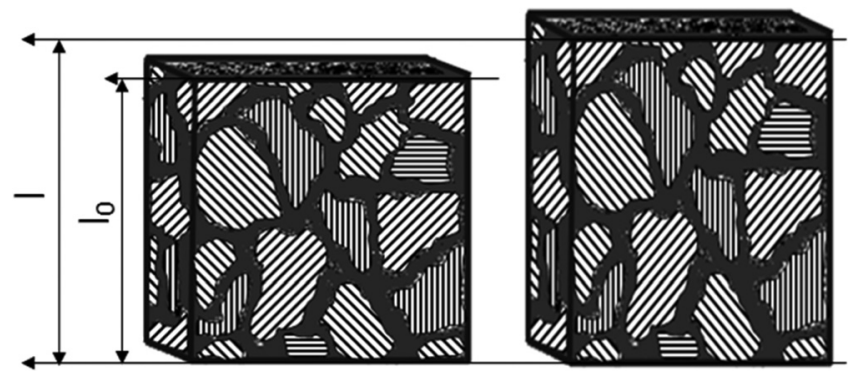

FIG. 1. Strain evolution during creep from (a) an undeformed state to (b) a deformed state under the action of a stress, $\sigma$. metals and develop subgrains, ${ }^{21}$ as a consequence of dislocation rearrangement in low-energy configurations. After grain boundaries have saturated (this corresponds to the last stages of the primary creep regime), the further evolution of subgrain structure (subgrain thickening) controls the deformation rate (Fig. 2). This substructure evolution follows the conventional scheme of heterogeneous transformations described by the JMAK model. Bulk nucleation of subgrains is described by an exponent $m=3$ (see Eq. (8), and Mittemeijer ${ }^{14}$ ), including growth and coalescence (subgrain boundary impingement). Subsequently, dislocations will accumulate until subgrain boundary saturation. In class-A alloys, the dislocation distribution during primary creep remains homogeneous forming a forest. Although no subgrains are formed during creep of these alloys, it is considered that the evolution of forests of dislocations during creep can be described using an equivalent sequence that of subgrains for the present purpose.

Finally, the strain due to dislocation movement saturates because (a) there is a finite quantity of dislocations that can be stored in the material, ${ }^{22}$ or (b) the stress field associated with the subgrain/forest structure increases until it cancels the effect of the external stress field, i.e., it exhausts the driving force for dislocation movement.

In summary, the two contributions to strain: (a) dislocations movement inside the grain boundary until grain boundary saturation and complete subgrain structure development in class-M alloys $(m \sim 1)$, and (b) subgrain boundary thickening up to subgrain saturation/forest saturation $(m \sim 3)$ can be described as

$$
\varepsilon=\varepsilon_{0}+A_{1}\left(1-e^{-A_{2} t}\right)+A_{3}\left(1-e^{-A_{4} t^{3}}\right),
$$

where $A_{1}$ and $A_{3}$ are strain terms that depend on stress and temperature and $A_{2}$ and $\left(A_{4}\right)^{1 / 3}$ are frequencies (analogous to reaction rates in isothermal transformations). Although damage always occurs along the whole creep curve, its contribution to deformation is considered negligible during subgrain formation and saturation.

It is further proposed that: (a) the primary creep region is dominated by dislocation movement inside the grain until complete subgrain formation, Figure 2 step (I) to step (III),

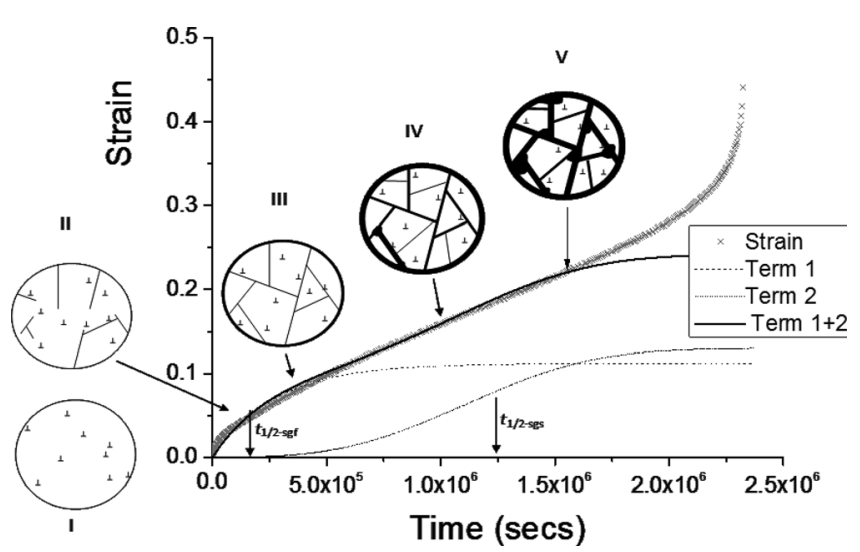

FIG. 2. Pictorial description of the microstructure evolution at different stages of the creep curve, based on experimental data of AA6061. The two terms of Eq. (11) as well as their sum are displayed. 
and (b) the secondary creep region is controlled by the storage of dislocations at subgrain boundaries, Figure 2 step (III) to step (V). The increase in creep strain rate associated with the tertiary stage is not controlled by diffusive process of dislocation movement, but rather by damage. ${ }^{23-25}$ Therefore, we will not consider tertiary creep in the SSTC model.

\section{PHYSICAL MEANING OF CREEP PARAMETERS IN SSTC MODEL}

\section{A. Strains as a function of microstructural parameters}

$A_{1}$ in Eq. (11) represents the total amount of strain accumulated until subgrain formation is complete (primary creep). Therefore, it is possible to describe it using the Orowan relationship ${ }^{6}$

$$
\varepsilon_{O r}=A_{1}=M^{-1} b \rho h(g) .
$$

The dislocation density involved in the phenomenon is given by the Taylor equation ${ }^{26}$

$$
\rho=\left(\frac{\sigma}{\alpha G b M}\right)^{2}
$$

where $\sigma$ is the applied stress during creep and $\alpha$ is a constant that depends mainly on the geometrical configuration of dislocations. The temperature dependence is introduced through the shear modulus $G$. The average distance $h(g)$ traveled by dislocations is equal to the subgrain size because dislocations will only travel the distance required to generate those subgrains (i.e., they will meet before they reach any grain boundary). The subgrain size is inversely proportional to the applied stress $\sigma .^{27}$

Similarly to $A_{1}, A_{3}$ represents the total amount of strain accumulated from complete subgrain formation until subgrain boundary saturation (secondary creep, note that this saturation corresponds to the stage when damage becomes dominant) and is also described by Eq. (12).

As the stress is kept constant during creep, and the amount of active Frank-Reed sources for mobile dislocations generation does not depend on the grain or subgrain structure, it is readily deduced that

$$
A_{3}=A_{1}=\frac{h(g)}{b M^{3}}\left(\frac{\sigma}{\alpha G}\right)^{2} .
$$

The mobile dislocation density is only a small fraction of the total dislocation density. The proportionality between mobile and total dislocation density shown in HendersonBrown $^{28}$ has been used to calculate the subgrain size using Eq. (14).

\section{B. Strain rates as a function of microstructural parameters}

The physical meaning of $A_{2}$ and $A_{4}$ parameters is related in the JMAK model to the frequency of atomic jumps involved in the diffusion mechanisms. In the present view, they are linked to the half-lives for completion of the processes of subgrain formation and subgrain boundary saturation (see Fig. 2):

$$
t_{1 / 2-s g f}=\frac{\ln 2}{A_{2}} \quad \text { and } \quad t_{1 / 2-s g s}=\left(\frac{\ln 2}{A_{4}}\right)^{1 / 3} .
$$

$A_{2}$ and $\left(A_{4}\right)^{1 / 3}$ can, therefore, be interpreted as deformation rates. We will call them characteristic deformation rates.

Since the deformation mechanism is the same before and after subgrain formation, the two parameters must be proportional to each other, i.e.,

$$
A_{4}=\left(a A_{2}\right)^{3} \text {. }
$$

While, at this point, this is just a reasonable hypothesis, we will see later that experimental data do support it.

During subgrain boundary saturation, a stress field around subgrain boundaries slows the dislocation movement down, while no hindrance is present when subgrains are still forming. In other words, the effective stress acting on moving dislocations during secondary creep decreases as the "reaction" proceeds. It is proposed that the factor $a$ in Eq. (16) quantifies the effect of the stress field on the dislocation motion (deformation rate). $a$ is in fact a measure of the decrease of creep rate due to the presence of subgrains. It depends on both material and experimental conditions. This dependence is at present unknown, and $a$ cannot be quantitatively related to microstructural parameters. It can be written as

$$
a^{3}=\frac{\ln 2}{(\ln 2)^{3}} \cdot\left(\frac{t_{1 / 2-s g f}}{t_{1 / 2-s g s}}\right)^{3}=2.08 \cdot\left(\frac{t_{1 / 2-s g f}}{t_{1 / 2-s g s}}\right)^{3} .
$$

In general, $a \in\left[0,2^{1 / 3}\right]$, and it must hold $a \ll 1$.

We can discuss two limit cases:

$a \rightarrow 0$ implies either that formation of subgrain boundaries is instantaneous $\left(t_{1 / 2 \text {-sgf }} \rightarrow 0\right)$ or there is no saturation of the subgrain boundaries $\left(t_{1 / 2 \text {-sgs }} \rightarrow \infty\right)$. The first case is unrealistic, while the second corresponds to materials with coarse grain structures, or undergoing a very low applied stress.

$a \rightarrow 2^{1 / 3}$ implies that subgrains form and subgrain boundaries saturate simultaneously; this occurs, for instance, in very small grain size materials, where the subgrain size coincides with the grain size. This is also known as the case of constant substructure creep. ${ }^{29}$

Based on the well-known equations of the "steady state" creep, Eqs. (1) and (2), it is possible to link $A_{2}$ and $A_{4}$ to microstructural parameters. In fact, deriving Eq. (11) with respect to time, we obtain

$$
\dot{\varepsilon}(t)=A_{1} A_{2} e^{-A_{2} t}+3 A_{3} A_{4} t^{2} e^{-A_{4} t^{3}} .
$$

From Eqs. (1) and (2), the minimum creep strain rate is described as

$$
\dot{\varepsilon}_{s s}=K\left(\frac{\sigma}{G}\right)^{n} e^{\frac{-Q_{c}}{R T}} .
$$

Therefore, equating expressions (17) at time $t_{\mathrm{m}}$ (time to reach the minimum strain rate) and (18), and using Eq. (14) 


$$
\varepsilon_{O r}\left(A_{2} e^{-A_{2} t_{m}}+3 A_{4} t_{m}^{2} e^{-A_{4} t_{m}{ }^{3}}\right)=K\left(\frac{\sigma}{G}\right)^{n} e^{\frac{-Q c}{R T}} .
$$

Considering Eq. (13), we find

$$
A_{2}\left(e^{-A_{2} t_{m}}+3 \cdot a^{3}\left(A_{2} t_{m}\right)^{2} e^{-\left(a A_{2} t_{m}\right)^{3}}\right)=\frac{K}{\varepsilon_{O r}}\left(\frac{\sigma}{G}\right)^{n} e^{\frac{-Q c}{R T}} .
$$

At this point, $t_{\mathrm{m}}$ is still unknown. However, as the strain rate is minimum at $t_{\mathrm{m}}$, deriving again Eq. (17) and considering Eqs. (14) and (16), we find

$$
\begin{aligned}
\ddot{\varepsilon}= & \varepsilon_{O r}\left[-A_{2}{ }^{2} e^{-A_{2} t_{m}}+6 a^{3} \cdot\left(A_{2}{ }^{3} t_{m}\right) e^{-\left(a A_{2} t_{m}\right)^{3}}\right. \\
& \left.-9 a^{6}\left(A_{2}{ }^{3} t_{m}{ }^{2}\right)^{2} e^{-\left(a A_{2} t_{m}\right)^{3}}\right]=0 .
\end{aligned}
$$

The solution of this transcendental equation can be found reorganizing it into two terms. If we define $x=A_{2} t_{m}$ and $a^{\prime}=3 a^{3}$, we can write

$$
e^{-x}=a^{\prime} x * e^{-\frac{d^{\prime} x^{3}}{3}}\left(2-a^{\prime} x^{3}\right) .
$$

The functions at the two sides of the equality are plotted in Figure 3.

There are two solutions to Eq. (22) for $x>0$, say, $x_{1}$ and $x_{2}>x_{1}$. They can be identified with two different strain rates. In fact, there are two known mechanisms controlling deformation during high temperature creep of metals: dislocation climb and dislocation glide. ${ }^{30,31}$ The creep strain rate (or the transformation reaction rate) will depend on the slowest between the controlling mechanisms. Therefore, $x_{1}$ (depending on $a$ ) must be chosen as the physically meaningful root of Eq. (22).

Inserting $x_{1}=A_{2} \times t_{\mathrm{m}}$ in Eq. (20) and taking into account Eq. (14), we find that

$$
A_{2}=\frac{b K(\alpha M)^{2}}{g \cdot l\left(a, x_{1}\right)}\left(\frac{\sigma}{G}\right)^{n-2} e^{\frac{-Q c}{R T}},
$$

where $l\left(a, x_{1}\right)=e^{-x_{1}}+3 \cdot a^{3} x_{1}^{2} e^{-\left(a x_{1}\right)^{3}}$.

Consequently, we obtain

$$
A_{4}=\left(a \frac{b K(\alpha M)^{2}}{g \cdot l\left(a, x_{1}\right)}\right)^{3}\left(\frac{\sigma}{G}\right)^{3(n-2)} e^{\frac{-3 Q c}{R T}},
$$

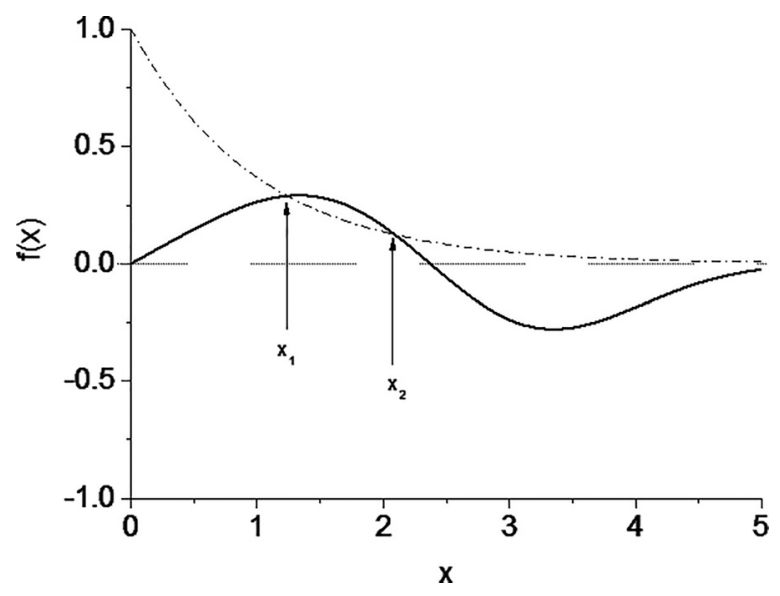

FIG. 3. Graphical solution of Equation (22) showing two roots for $x>0$. and

$$
t_{m}=\frac{x_{1} \cdot g \cdot l\left(a, x_{1}\right)}{b K(\alpha M)^{2}}\left(\frac{\sigma}{G}\right)^{2-n} e^{\frac{Q c}{R T}}
$$

The creep curve in the primary and secondary stages can be described as

$$
\begin{aligned}
\varepsilon= & \frac{h(g)}{b(\alpha M)^{2}}\left(\frac{\sigma}{G}\right)^{2}\left[\left(1-e^{-\frac{b K(\alpha M)^{2}}{g \cdot\left(\alpha, x_{1}\right)}\left(\frac{\sigma}{G}\right)^{n-2} e^{\frac{-Q c}{R T}} t}\right)\right. \\
& +\left(1-e^{\left.\left.-\left(a \frac{b K(\alpha M)^{2}}{g \cdot\left(a, x_{1}\right)}\right)^{3}\left(\frac{\sigma}{G}\right)^{3(n-2)} e^{\frac{-3 Q c}{R T} t^{3}}\right)\right] .}\right.
\end{aligned}
$$

The SSTC model allows predicting the creep behavior of the alloy on the basis of microstructural and mechanical properties, as well as on experimental conditions. The sole parameter $a$ needs to be experimentally determined. The microscopic creep mechanism is related to the $n$ parameter in Equation (26) in the same way as it was described by Sherby, ${ }^{5}$ Equation (1). Moreover, the transitions between creep mechanisms with the applied stress ${ }^{32,33}$ are considered in the model in the same manner as in the classical steady state power law creep description.

\section{EXPERIMENTAL CREEP CURVES}

The creep behavior of ingot metallurgy commercially pure aluminum (99.8\%) and AA6061 alloy was studied by means of stress-controlled tensile creep tests at 573 and $623 \mathrm{~K}$ and applied stresses between 2.7 and 16.1 MPa (see Table II). A heating rate of $100 \mathrm{~K} / \mathrm{h}$ until the creep testing temperature was applied, following previous works. ${ }^{30}$ This is equivalent to a heat treatment prior to sample loading and provokes an averaged precipitation state in the material. Constant stress during sample elongation was guaranteed by means of an Andrade's cam, which reduces the applied load according with the sample section reduction. Cylindrical samples, with threaded heads and a gauge region of $3 \mathrm{~mm}$ diameter and $10 \mathrm{~mm}$ length, were machined. The tensile axis was parallel to the extrusion axis of the samples. The elongation and the applied load as a function of time were recorded by two linear variable differential transducers, LVDTs and a load cell, respectively. A clamping system was developed inhouse, in order to suppress the contribution of the machine and grip elongation to the total strain. This allows obtaining the actual sample creep deformation with time.

Samples were deformed until failure or until the maximum displacement of the Andrade's arm was reached (which corresponds to $\varepsilon=0.55$ in the present case). Selected plots of strain vs. time are shown in Figure 4.

\section{MODEL RESULTS: DETERMINATION OF MICROSTRUCTURAL PARAMETERS}

We proceed the following way: we first fit the experimental creep curves considering $A_{1}$ to $A_{4}$ as fitting parameters. This approach will allow us to (a) determine the unknown parameter $a$ and (b) extract some relevant 
TABLE II. Results from fitting procedure using Eq. (11), as well as test conditions for the $99.8 \% \mathrm{Al}$ and AA6061 alloy.

\begin{tabular}{lcccccccc}
\hline \hline Material & Stress (MPa) & $\dot{\varepsilon}_{S S}\left(\mathrm{~s}^{-1}\right)$ & $\mathrm{T}(\mathrm{K})$ & Total strain & $\mathrm{A}_{1}=\varepsilon_{\mathrm{OR}}$ & $\mathrm{A}_{2}$ & $\mathrm{~A}_{3}=\varepsilon_{\mathrm{OR}}$ & $\mathrm{A}_{4}$ \\
\hline A1 99.8\% & 2.1 & $8.83 \times 10^{-9}$ & 573 & 0.55 & 0.028 & $8.64 \times 10^{-5}$ & 0.070 & $3.89 \times 10^{-15}$ \\
A1 99.8\% & 5.2 & $1.23 \times 10^{-7}$ & 573 & 0.56 & 0.150 & $8.62 \times 10^{-7}$ & 0.081 & $9.62 \times 10^{-19}$ \\
A1 99.8\% & 3.9 & $1.66 \times 10^{-6}$ & 623 & 0.55 & 0.258 & $1.31 \times 10^{-6}$ & 0.300 & $8.62 \times 10^{-17}$ \\
A1 99.8\% & 7.7 & $4.85 \times 10^{-5}$ & 623 & 0.53 & 0.261 & $2.91 \times 10^{-4}$ & 0.289 & $2.30 \times 10^{-12}$ \\
A1 99.8\% & 8.9 & $8.93 \times 10^{-5}$ & 623 & 0.50 & 0.352 & $4.76 \times 10^{-4}$ & 0.336 & $6.71 \times 10^{-12}$ \\
AA6061 & 9.2 & $1.10 \times 10^{-7}$ & 623 & 0.54 & 0.127 & $2.30 \times 10^{-6}$ & 0.163 & $2.30 \times 10^{-19}$ \\
AA6061 & 10.3 & $1.00 \times 10^{-7}$ & 623 & 0.08 & 0.039 & $8.93 \times 10^{-6}$ & 0.050 & $6.76 \times 10^{-18}$ \\
AA6061 & 12.0 & $4.20 \times 10^{-7}$ & 623 & 0.14 & 0.100 & $4.78 \times 10^{-6}$ & 0.090 & $3.53 \times 10^{-17}$ \\
AA6061 & 14.8 & $1.70 \times 10^{-6}$ & 623 & 0.55 & 0.200 & $1.33 \times 10^{-5}$ & 0.237 & $8.85 \times 10^{-15}$ \\
AA6061 & 16.1 & $6.20 \times 10^{-6}$ & 623 & 0.51 & 0.200 & $3.50 \times 10^{-5}$ & 0.253 & $8.85 \times 10^{-14}$ \\
\hline \hline
\end{tabular}

microstructural parameter contained in Eq. (26), such as the subgrain size. In fact, the subgrain size strongly depends on the experimental conditions and can only be measured postmortem.

An example of creep curve fitting made using Eq. (11) is shown in Figure 5.

The parameters $A_{i}(i=1-4)$ extracted from the fit of all available data are shown in Table II.

The analysis of the parameters $A_{1}$ to $A_{4}$ confirms that they are not independent from each other.

As predicted by the model, it holds $A_{1} \approx A_{3}$ (Figure 6). This means that the total strains due to subgrain formation and subgrain boundary saturation are indeed the same.
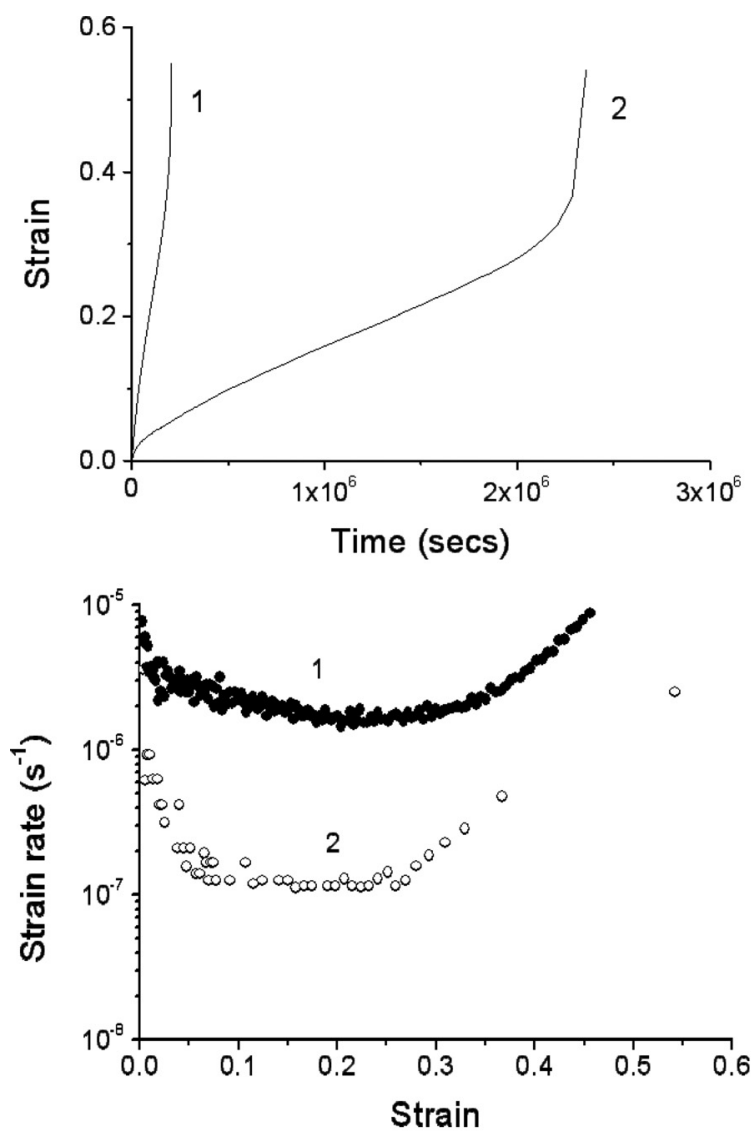

(a)
We represent in Fig. 7 all data of $99.8 \% \mathrm{Al}$ and AA6061 alloy confounded, plotting the curve $A_{4}=\left(a \times A_{2}\right)^{c}$. We find a linear relationship between $A_{2}$ and $A_{4}$ (Figure 7). The exponent of the fitting curve is $c=2.45 \pm 0.42$, and we obtain $a=0.05 \pm 0.05$. In Figure 7, a line with slope $c=3$ is also displayed. We can observe that the model curve $(c=3)$ lies very near to the experimental data, and therefore, we can trust the obtained value of $a$ as a reasonable estimation of the parameter in Eq. (22).

The experimentally determined proportionality between $A_{4}$ and $A_{2}$ confirms the hypothesis made above: that the mechanisms for deformation during subgrain formation and subgrain boundary saturation are basically the same:
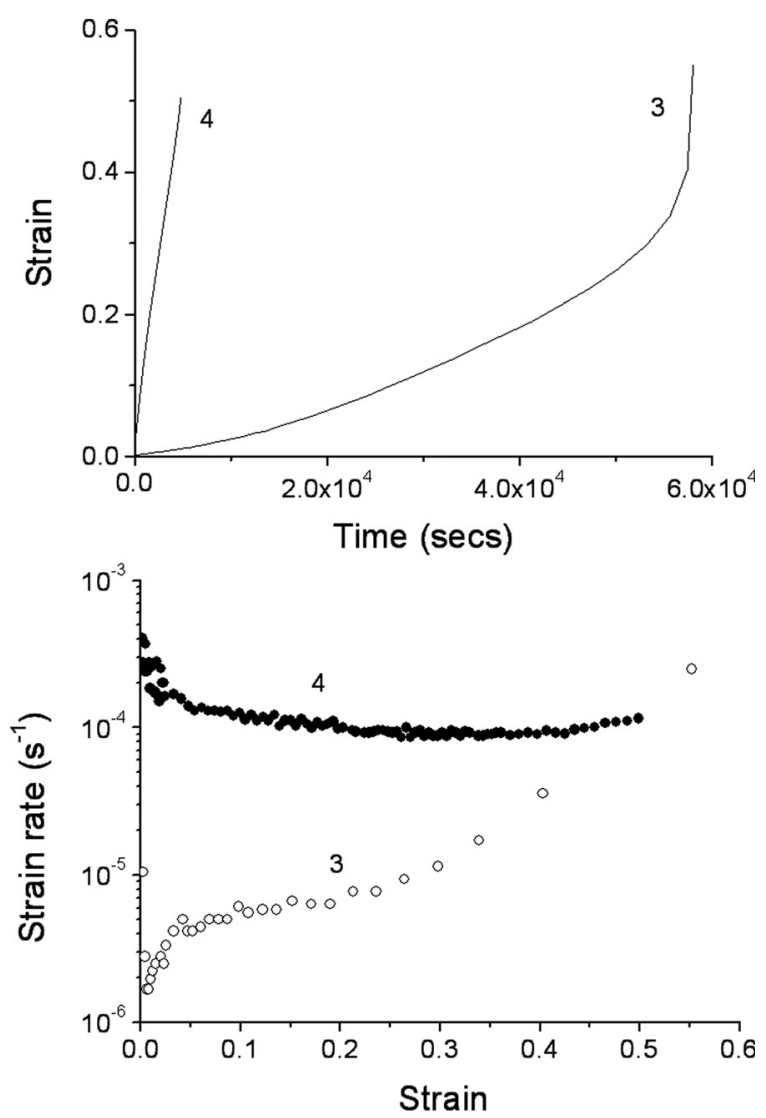

(b)

FIG. 4. Selected creep curves (a) 99.8\% Al: (1) $3.9 \mathrm{MPa}$ and $573 \mathrm{~K}$ and (2) $9 \mathrm{MPa}$ and $623 \mathrm{~K}$. (b) AA6061 alloy: (3) $9.2 \mathrm{MPa}$ and $623 \mathrm{~K}$ and (4) $16.1 \mathrm{MPa}$ and $623 \mathrm{~K}$. Both $\varepsilon$ vs. $t$ and $\mathrm{d} \varepsilon / \mathrm{d} t$ vs. $\varepsilon$ are shown for the same sets of data. 


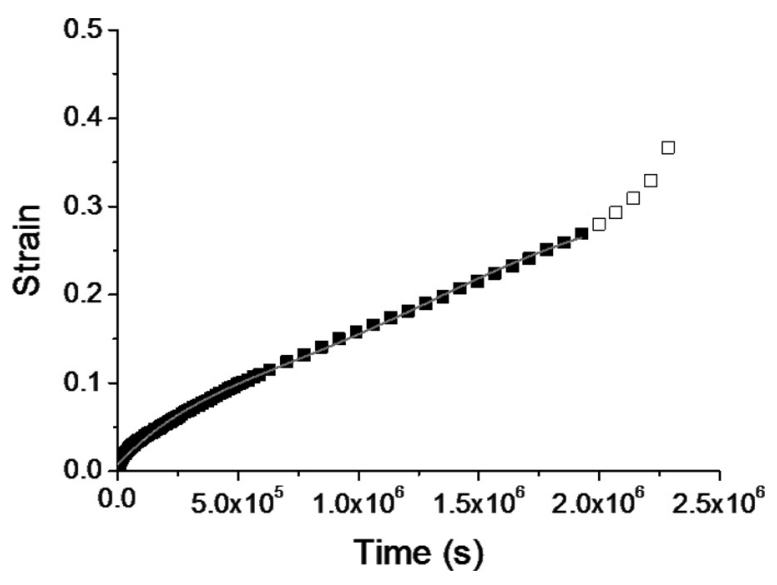

FIG. 5. Creep curve fitting using the SSCT model described in Eq. (11). Open squares are not used for the fit.

diffusion controlled dislocation motion. The functional dependences shown in Figures 6 and 7 can be considered as a validation of our model.

The model set above also allows us to determine the subgrain size, using Eq. (14) and the input parameters listed in Table III. The results are given in Table II. The obtained subgrain size reasonably agrees with the microstructure of the $99.8 \% \mathrm{Al}$ and the AA6061 alloy, shown in Fig. 8 and with data reported in Blum ${ }^{6}$ and Nakai. ${ }^{34}$

\section{TERTIARY CREEP STAGE}

The secondary creep regime terminates once the stress field generated by the saturated subgrain boundaries prevents the dislocation movement. This internal stress field also promotes material damage evolution during the tertiary creep regime. Although tertiary creep is outside the scope of this work, we now discuss how to incorporate existing damage models into the above developed scheme, to include tertiary creep in our description.

Creep damage is manifested by the formation and growth of creep voids or cavities from tenths to tens of microns. ${ }^{35,36}$ Although cavities appear since the early stages of creep $^{37}$ or can be present even prior to creep testing, ${ }^{38}$ they dominate the strain rate only after the onset of the

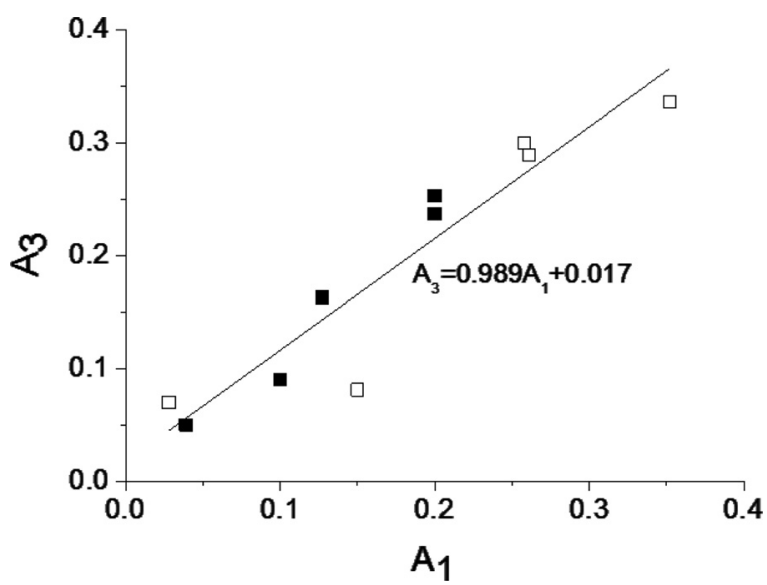

FIG. 6. Linear relationship between $A_{1}$ vs. $A_{3}$ parameters of Eq. (11) (open squares $\mathrm{Al}$ 99.8\%). The solid line indicates fitting curve.

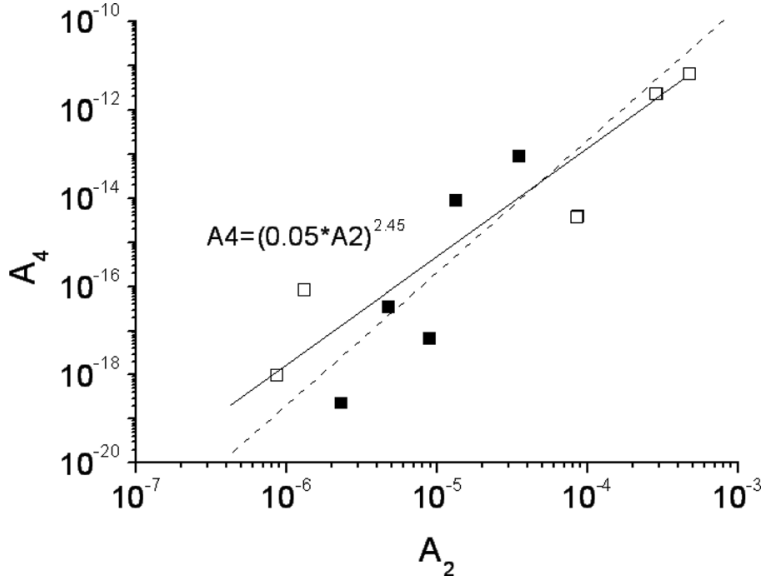

FIG. 7. Relationship between $A_{4}$ vs. $\left(A_{2}\right)^{3}$ parameters of Eq. (11) (open squares $\mathrm{Al}$ 99.8\%). The solid line indicates fitting curve, and black dashed line represents a slope of 3 curve.

tertiary stage of creep. Finally, cavity coalescence form grain boundary cracks, which then propagate to cause failure.

In order to explain the rupture time under creep conditions, Kachanov ${ }^{24}$ has postulated a power law dependence (on applied uniaxial stress) of a damage function. This dependence was linked to creep strain rate by Rabotnov ${ }^{39}$ and further extended to multiaxial stress states by Leckie. ${ }^{40}$ The relationship reads

$$
\dot{\varepsilon} \propto \frac{\sigma^{t}}{(1-w)^{s}},
$$

where $w$ is a damage function, and $s$ and $t$ are constants.

Equation (27) is the most common way to introduce the effect of creep damage on the strain rate. It seems reasonable to insert an additional term similar to the right hand side of Eq. (27) into Eq. (22) in order to include creep damage and describe tertiary creep.

\section{SUMMARY AND CONCLUSIONS}

A view of primary and secondary creep of metals is proposed based on isothermal transformation processes (described by the JMAK model). Physically, this is justified by the fact that creep is a diffusion-controlled process. We used experimental creep curves of pure aluminum $(99.8 \%)$ and AA6061 alloy to validate the proposed model. The main conclusions of this work are as follows:

TABLE III. Parameters used in the calculation of the subgrain size.

\begin{tabular}{lcc}
\hline \hline Parameter & Definition & Value (Units) \\
\hline$\alpha$ & Constant & 0.3 \\
$G$ & Shear modulus & $21800(\mathrm{MPa}) @ 573 \mathrm{~K}$ \\
& & $20900(\mathrm{MPa}) @ 623 \mathrm{~K}$ \\
$b$ & Burgers vector & $2.86 \times 10^{-10}(\mathrm{~m})$ \\
$M$ & Taylor factor & 3.06 \\
$D$ & Diffusion coefficient & $1.7 \times 10^{-4} \mathrm{e}^{(-142000 / \mathrm{RT})}\left(\mathrm{m}^{2} \mathrm{~s}^{-1}\right)$ \\
$R$ & $D=D_{0} \cdot \exp \left(\frac{-Q_{d}}{R T}\right)$ & $8.314(\mathrm{~J} / \mathrm{K} \mathrm{mol})$ \\
$k$ & Gas constant & $1.38 \times 10^{-23}(\mathrm{~J} / \mathrm{K})$ \\
\hline \hline
\end{tabular}




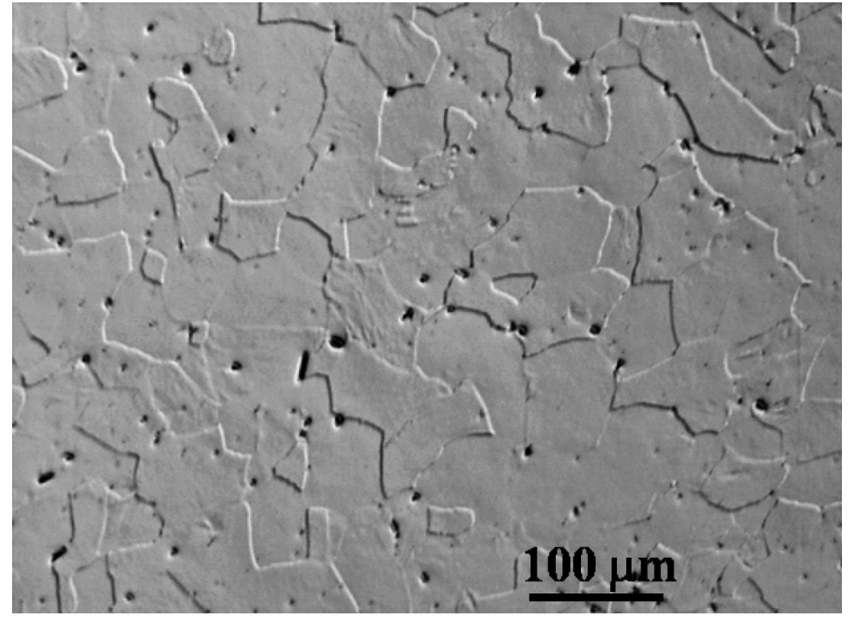

(a)

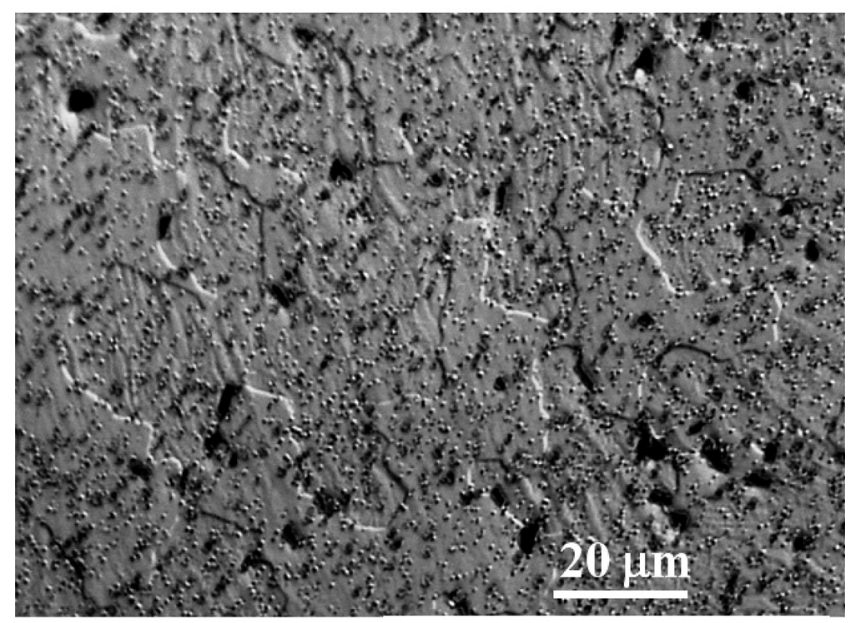

(b)

FIG. 8. Typical microstructure of the $99.8 \% \mathrm{Al}$ (a) and the AA6061 alloy (b).

(1) Creep in metals can be described by accumulation of deformation "quanta," given by the Orowan relation. Those "quanta" are related to the minimum distance travelled by a dislocation (the Burger's vector $b$ ).

(2) The collective dislocation motion first creates subgrains in class-M alloys and then saturates their boundaries. In class-A alloys, a similar evolution of dislocations structures but arranged in forests is assumed.

(3) The deformation associated with subgrain formation and subgrain saturation describes creep curves until damage becomes dominant (tertiary creep). In this framework, primary and secondary creeps are described in a unified way.

(4) Experimental data on $99.8 \% \mathrm{Al}$ and commercial AA6061 alloy fully validate the model. It is proven that (a) the total deformations associated with subgrain formation and saturation coincide; this reflects the fact that the average distance travelled by a dislocation is basically the subgrain size; (b) the characteristic deformation rates associated with subgrain formation and saturation are proportional, i.e., the deformation mechanism is the same before and after subgrain formation, but dislocations move under a reduced equivalent stress after subgrain formation.

\section{ACKNOWLEDGMENTS}

This paper was funded by Project No. MAT201455415-C3-1-R from MINECO, Spain. The authors are thankful to Rüdiger Jaenisch for useful discussion. BAM is acknowledged for travel support.

${ }^{1}$ R. W. Evans, J. D. Parker, and B. Wilshire, Int. J. Pressure Vessels Piping 50, 147-160 (1992).

${ }^{2}$ P. Krizik, M. Balog, M. Nosko, M. V. C. Riglos, J. Dvorak, and O. Bajana, Mater. Sci. Eng. A 657, 6-14 (2016).

${ }^{3}$ V. Dudko, A. Belyakov, and R. Kaibyshev, Trans. Indian Inst. Met. 69(2), 223-227 (2016)

${ }^{4}$ A. Alizadeh, A. Abdollahi, and H. Biukani, J. Alloys Compd. 650, 783-793 (2015)

${ }^{5}$ B. Walser and O. D. Sherby, Scr. Metall. 16, 213-219 (1982).

${ }^{6} \mathrm{~W}$. Blum, in Creep of Aluminium and Aluminium Alloys. Hot Deformation of Aluminium Alloys, edited by T. G. Langdon, H. D. Merchant, J. G. Morris, and M. A. Zaidi (The Minerals, Metals \& Materials Society, Warrendale, PA, USA, 1991), pp. 181-209.

${ }^{7}$ O. D. Sherby and P. Burke, Prog. Mater. Sci. 13, 323-390 (1968).

${ }^{8} \mathrm{~F}$. Garofalo, Fundamentals of Creep and Creep Rupture in Metals (Macmillan, New York, 1965).

${ }^{9}$ B. Wilshire and R. W. Evans, Creep Behavior of Crystalline Solids (Pineridge Press, Swansea, 1985).

${ }^{10}$ W. D. Day and A. P. Gordon, in Proceedings of the ASME Turbo Expo 2013: Turbine Technical Conference and Exposition, San Antonio, Texas, USA (2013), Volume 7A: Structures and Dynamics, p. V07AT26A002.

${ }^{11}$ H. Mecking and U. F. Kocks, Acta Metal. 29, 1865-1875 (1981).

${ }^{12}$ Y. Estrin, Mater. Sci. Eng. A 463, 171-176 (2007).

${ }^{13} \mathrm{M}$. Rakotondrabe, in Proceedings of the IEEE International Conference on Robotics and Automation, ICRA, St. Paul, Minnesota, USA (2012), pp. 4577-4581.

${ }^{14}$ E. J. Mittemeijer, J. Mater. Sci. 27, 3977-3987 (1992).

${ }^{15}$ S. Jun, H. Zhang, and J. Bechhoefer, Phys. Rev. E 71, 011908 (2005).

${ }^{16}$ C. P. Yang and J. F. Nagle, Phys. Rev. A 37(10), 3993-4000 (1988).

${ }^{17}$ T. Huang, T. Tsuji, M. R. Kamal, and A. D. Rey, Phys. Rev. E 58, 7507-7516 (1998).

${ }^{18}$ M. Fanfoni and M. Tomellini, Nuovo Cimento Soc. Ital. Fis., D 20(7-9), 1171-1182 (1998).

${ }^{19} \mathrm{G}$. Korniss and T. Caraco, J. Theor. Biol. 233, 137-150 (2005).

${ }^{20}$ B. Kämpfer, Ann. Phys. (Leipzig) 9, 605-635 (2000).

${ }^{21}$ S.-W. Nam, D.-H. Lee, and K. T. Hong, in Proceedings of the Fifth Conference on Creep of Metals, edited by D. A. Woodford, C. H. A. Townley, and M. Ohnami, Lake Buena Vista, Florida, USA (1992), pp. 18-21.

${ }^{22}$ A. Borrego, Ph.D. thesis, Complutense University of Madrid, 2003.

${ }^{23}$ M. Vose, B. Fedelicha, F. Otto, and G. Eggeler, Proc. Mater. Sci. 3, 21-26 (2014).

${ }^{24}$ L. M. Kachanov, Int. J. Fract. 97, xi-xviii (1999).

${ }^{25}$ B. Camin, Ph.D. thesis, Technischen Universität Berlin, 2014.

${ }^{26}$ U. F. Kocks and H. Mecking, Prog. Mater. Sci. 48, 171-273 (2003).

${ }^{27}$ J. Ibañez, J. Castellanos, and G. Gonzalez-Doncel, Mater. Trans. JIM 37(1), 63-66 (1996).

${ }^{28} \mathrm{M}$. Henderson-Brown and K. F. Hale, High Voltage Electron Microscopy, edited by P. R. Swarm, C. J. Humphreys, and M. J. Goringe (Academic Press, London, 1974), p. 206.

${ }^{29}$ O. D. Sherby, R. H. Klunt, and A. K. Miller, Met. Trans. A 8, 843-850 (1977).

${ }^{30}$ R. Fernández and G. Gonzalez-Doncel, J. Alloys Compd. 440, 158-167 (2007).

${ }^{31}$ R. Fernández and G. Gonzalez-Doncel, Mater. Sci. Eng. A 550, 320-324 (2012).

${ }^{32}$ Y-J. Wang, A. Ishii, and S. Ogata, Phys. Rev. B 84, 224102 (2011).

${ }^{33}$ X.-S. Yang, Y.-J. Wang, H.-R. Zhai, G.-Y. Wang, Y.-J. Su, L. H. Dai, S. Ogata, and T.-Y. Zhang, J. Mech. Phys. Sol. 94, 191-206 (2016).

${ }^{34}$ M. Nakai and G. Itoh. Mater. Trans. 55(1), 114-119 (2014).

${ }^{35}$ P. J. Bouchard, P. J. Withers, S. A. McDonald, and R. K. Heenan, Acta Mater. 52(1), 23-34 (2004).

${ }^{36}$ R. W. Ballufi and L. L. Seigle, Acta Metall. 5(8), 449-454 (1957). 
${ }^{37}$ H. Toda, Z. A. B. Shamsudin, K. Shimizu, K. Uesugi, A. Takeuchi, Y. Suzuki, M. Nakazawa, Y. Aoki, and M. Kobayashi, Acta Mater. 61, 2403-2413 (2013).

${ }^{38}$ M. E. Kassner and T. A. Hayes, Int. J. Plast. 19, 1715-1748 (2003).
${ }^{39}$ Y. N. Rabotnov, Creep Problems of Structural Members, North-Holland Series in Applied Mathematics and Mechanics (North-Holland Publishing Company, Amsterdam/London, 1969).

${ }^{40}$ F. A. Leckie and D. R. Hayhurst, Acta Metal. 25, 1059-1070 (1977). 